\title{
Plexiform Neurofibroma of the Larynx: A Challenging Management Dilemma
}

Nupur Kapoor Nerurkar, Gauri Kapre

\begin{abstract}
Introduction: Among the many causes responsible for stridor in the pediatric population, tumors of the larynx are relatively rare. Rarer still is the presence of endolaryngeal neurofibromas. Plexiform neurofibromas are associated with type I neurofibromatosis (NF1). Their typical characteristic is that they are diffuse tumors with indistinct margins, which makes complete resection a challenge and the chances of recurrences higher.
\end{abstract}

Objective: To document our experience with endolaryngeal neurofibromas and to discuss the treatment options available for this rare condition.

Methods: We present two cases of plexiform neurofibromas in pediatric patients. Both children presented with large supraglottic masses which interfered with breathing and swallowing. They also fulfilled the criteria for type I neurofibroma. Endolaryngeal approach with microdebrider and laser-assisted surgical excision was performed in both cases.

Result: Following surgery, both patients had uneventful recovery and no subsequent breathing or swallowing difficulties.

Conclusion: The dilemma in the management of endolaryngeal neurofibroma is the choice between endolaryngeal laser and aggressive external approach surgery. A short review of the limited existing literature shows that it is wiser to limit the surgery to as complete a resection as is possible endoscopically.

Keywords: Larynx, Plexiform neurofibroma, Surgery.

How to cite this article: Nerurkar NK, Kapre G. Plexiform Neurofibroma of the Larynx: A Challenging Management Dilemma. Int J Phonosurg Laryngol 2013;3(2):55-57.

Source of support: Nil

Conflict of interest: None declared

\section{INTRODUCTION}

Pediatric airway obstruction can arise from a variety of causes; the commonest being croup, laryngomalacia, subglottic stenosis and foreign body aspiration. However, benign laryngeal tumors leading to airway obstruction is not an entirely uncommon situation. Among the benign laryngeal tumors, neurofibroma is a relatively rare cause of pediatric stridor. Neurofibromatosis is classified as type 1 and 2. Type 1 is neurofibromatosis (NF1) also known as Von Recklinghausen's disease in which plexiform neurofibromas may occur. ${ }^{1}$ We present here two case studies of supraglottic plexiform neurofibroma of the larynx in children causing airway obstruction along with a review of literature.

\section{CASE REPORTS}

\section{Case 1}

A 3 years old male child presented to a pediatric hospital with difficulty in breathing for which an emergency tracheostomy had to be performed. The child was then referred to our center for further definitive management. A CT scan of the larynx was performed. This revealed a large supraglottic mass arising from the right lateral border of the epiglottis, aryepiglottic fold, arytenoid cartilage and the medial border of the pyriform fossa. The base of the tongue and vallecula were free from the tumor and the vocal cords were uninvolved. Contrast-enhanced view showed the mass to be iso to hyperintense.

We decided to first perform a microlaryngoscopic (MLS) examination under general anesthesia. There was a large rounded swelling occupying the entire supraglottic larynx on the right side, extending from the epiglottis to the arytenoid cartilage (Fig. 1). An incisional biopsy of this lesion was performed using a $\mathrm{CO}_{2}$ laser with the acublade. Telescopic examination revealed normal glottis and subglottis. The histopathological examination of the specimen was reported as 'plexiform neurofibroma'.

A thorough clinical examination for signs of NF1 was done in collaboration with a pediatric ophthalmologist, orthopedic surgeon and pediatric surgeon. The child had obvious café-au-lait spots on his lower back (total 7 in number), with the largest one being around $4 \mathrm{~cm}$ in diameter (Fig. 2). The ophthalmic evaluation ruled out the presence of Lisch nodules or optic gliomas. There were no dysplastic changes in the long bones. The presence of two criteria ${ }^{2,3}$ (café-au-lait spots and plexiform neurofibroma) classified the case as a type 1 neurofibroma. There were no signs of neurofibromatosis in the parents or first degree relatives of the patient.

After getting a precise idea about the extent of the lesion and histopathological diagnosis, we decided to go ahead with a second surgery aiming for as complete an excision as possible. Under general anesthesia, with suspension microlaryngoscopy, a tricut $3.5 \mathrm{~mm}$ laryngeal microdebrider was used to debulk the lesion. After debulking the mass, the attachments of the tumor over the medial wall of the pyriform fossa and the false vocal fold on the right side were severed with the help of $\mathrm{a} \mathrm{CO}_{2}$ laser. A near complete excision was thereby achieved (Fig. 3). We inserted an infant feeding tube to facilitate nutrition during the postoperative period. 


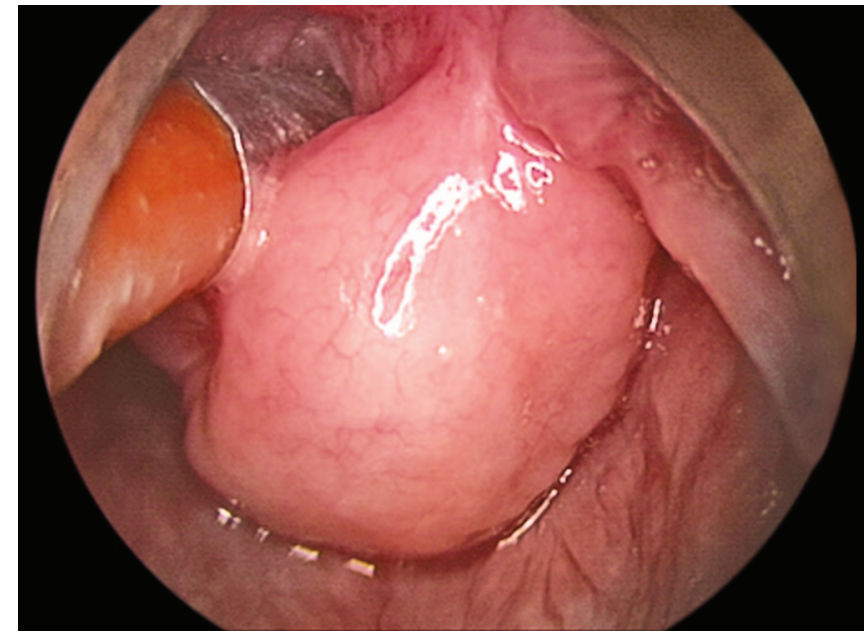

Fig. 1: Large smooth mass over the right aryepiglottic folds, false cords and arytenoid

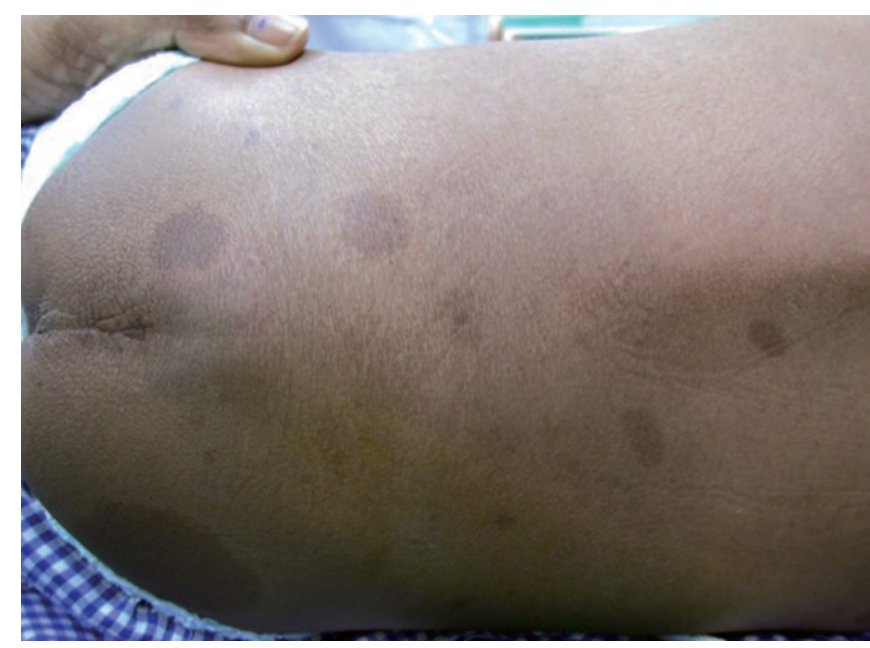

Fig. 2: Multiple café-au-lait spots over the back

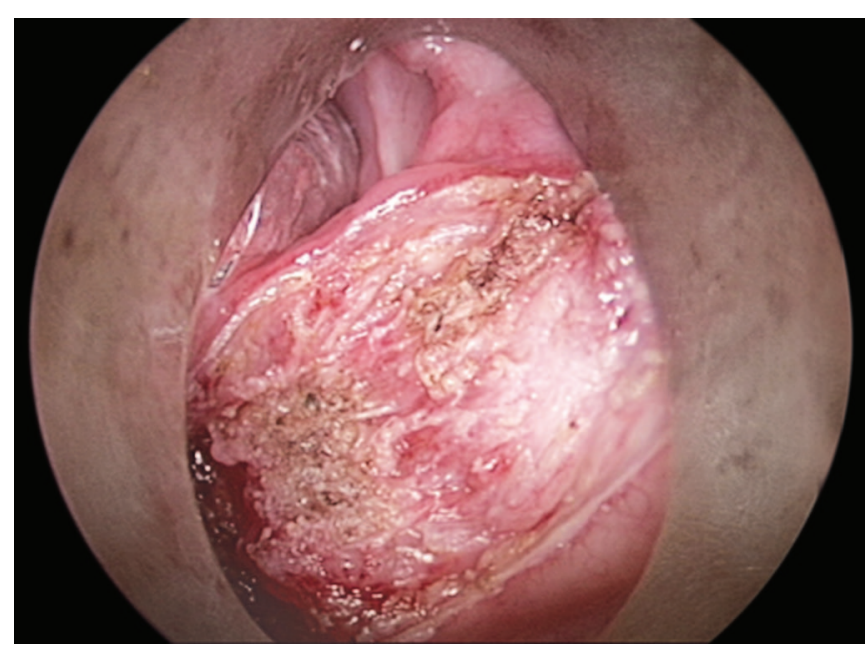

Fig. 3: Tumor excised with laser

A week later, the child was again taken under general anesthesia and the tumor bed was examined. Crusts and slough in the area were cleaned up and absence of any residual mass was confirmed (Fig. 4). Flexible laryngoscopic

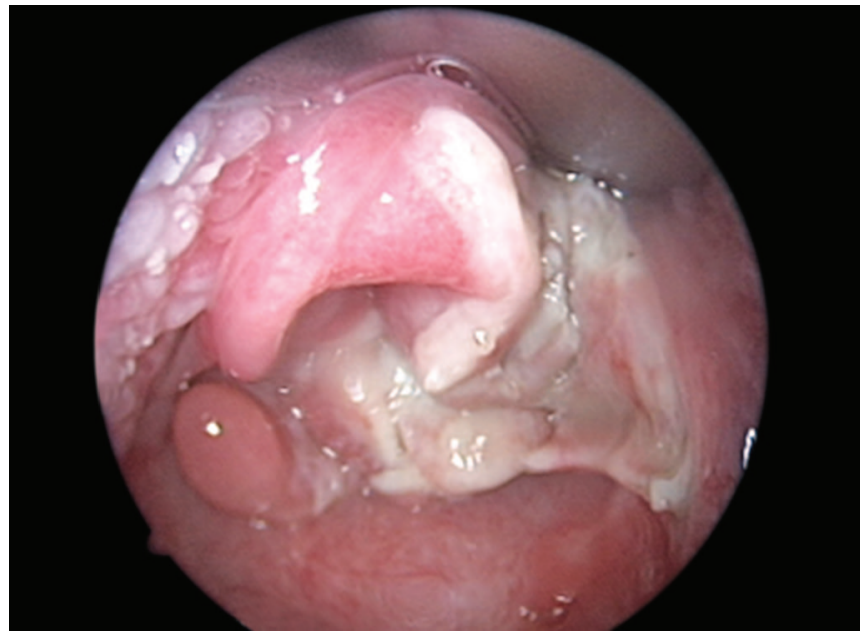

Fig. 4: Tumor bed one week postoperatively

evaluation 3 months following the surgery revealed healthy mucosa. The child is presently tolerating a full closure of the tracheostomy tube and has no difficulty with oral feeds.

\section{Case 2}

A 4 years old male child was referred to our center with history of mild dysphagia and failure to thrive. The child presented with a 'hot potato' speech, but there was no stridor. Flexible laryngoscopy revealed a large smooth surfaced mass involving the right aryepiglottic fold, false vocal fold, medial wall of the pyriform fossa, arytenoid cartilage and filling up the postcricoid space. A contrast-enhanced CT scan revealed an ill-defined soft tissue mildly enhancing mass in the supra glottis involving the right arytenoid, aryepiglottic fold, false cord and medial wall of the pyriform sinus.

The child was taken up for ML scopy under general anesthesia with laser safety protocol measures. As in the previous case, we performed an incisional biopsy using the AcuBlade system. Following the histopathological report of plexiform neurofibromatosis (Fig. 5), a completion surgery was performed a week later. The mass was excised from over the right aryepiglottic fold, right false vocal fold, arytenoid and medial wall of the pyriform fossa. The true vocal folds and subglottis were uninvolved.

The child had multiple café-au-lait spots over the body with kyphoscoliosis and sternal deformity. There were no Lisch nodules and no peripheral neuromas. There was no evidence of neurofibromatosis in first degree relatives.

\section{DISCUSSION}

The first case of an endolaryngeal neurofibroma was reported by Suchanek in $1925 .{ }^{4}$ The neurofibromas in NF1 could be plexiform (PN) or nonplexiform. Plexiform is generally congenital and, hence, present early in life. They are diffuse and ill-localised and thought to involve terminal fibers of multiple nerve branches. These are histologically 


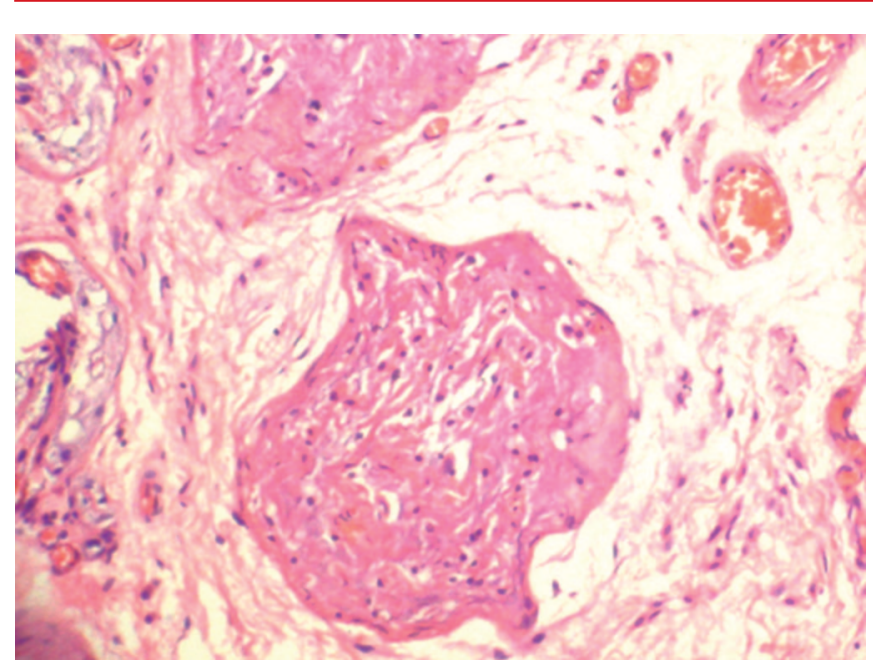

Fig. 5: Histopathological appearance of plexiform neurofibroma

distinct tumors showing intertwined spindle-shaped cells with dense surrounding connective tissue. Potential for malignant transformation has been reported at around $5 \% .{ }^{5}$ Needle et $\mathrm{al}^{6}$ reported the largest series of surgically managed plexiform neurofibromas and their series showed $54 \%$ recurrence within a 10 -year follow-up period with the greatest risk of recurrence being for head and neck neurofibromas and in children less than 10 years of age. They are locally infiltrating and complete resection is considered difficult. ${ }^{7}$ The usual site of occurrence of a laryngeal neurofibroma is the supraglottis; probable cause being the abundance of terminal nerve plexuses of the superior laryngeal nerve. ${ }^{8}$ These tumors are usually seen as large, smooth, submucosal masses located in the supraglottis resulting in difficulty in breathing and swallowing. These have been the presentation in most reported pediatric laryngeal neurofibromas. Most authors advocate that one must stay conservative while managing these tumors as they are always associated with a high risk of recurrence. Whenever surgical excision is being contemplated, one should aim at as complete an excision as possible. Endolaryngeal $\mathrm{CO}_{2}$ laser (as used in our case) is appropriate for medium-sized tumors. However, quite often, larger tumors might not be amenable to complete endolaryngeal resection. In such cases, external approaches, like lateral pharyngotomy or thyrotomy, have been described. ${ }^{9}$

\section{CONCLUSION}

Plexiform neurofibromas of the larynx are uncommon tumors with special predisposition for the supraglottic larynx. Initial devascularization incision with a laser followed by microdebrider-assisted debulking of the tumor mass, is what we recommend for optimum results. Due to the diffuse involvement of surrounding tissue, complete excision may be a challenge and long-term follow-up will be required to counter the risk of recurrence and potential malignant transformation.

\section{SUMMARY}

- Plexiform neurofibromas and endolaryngeal neurofibromas as such, are rare tumors.

- Complete excision is quite often difficult due to the diffuse nature of the tumor and rate of recurrences is quite high.

- There is some debate over endolaryngeal vs external approach for excision.

- The authors are of the opinion that endolaryngeal excision (with microdebrider and $\mathrm{CO}_{2}$ laser) should be preferred, attempting to remove as much tumor as possible with the aim to relieve the patient of his symptoms, rather than attempting complete excision by lateral pharyngotomy and producing morbidities or complications in the process.

\section{DECLARATION}

This paper had been presented as a poster by Dr Nerurkar at Laryngology (2011) in Malaysia.

\section{REFERENCES}

1. Wise JB, Cryer JE, Belasco JB, et al. Management of Head and Neck Plexiform Neurofibromas in Pediatric Patients with Neurofibromatosis Type 1. Arch Otolaryngol Head Neck Surg 2005;131:712-718.

2. Gutman DH, Aysworth A, Carey JC, et al. Diagnostic evaluation of multidisciplinary management of neurofibromatosis 1 and neurofibromatosis 2. JAMA 1997;278:51-57.

3. Rahbar R, Litrovnik BG, Vargas SO, et al. The Biology and Management of Laryngeal Neurofibroma. Arch Otolaryngol Head Neck Surg 2004;130:1400-1406.

4. Dave SP, Farooq U, Civantos FJ. Management of advanced laryngeal and hypopharyngeal plexiform neurofibroma in adults. Am J Otolaryngol Head and Neck Medicine and Surgery 2008;29:279-283.

5. Ducatman BS, Scheithauer BW, Piepgras DG, et al. Malignant nerve sheath tumors. Cancer 1986;57:2006-2021.

6. Needle MN, Cnaan A, Datillo J, et al. Prognostic signs in the surgical management of plexiform neurofibroma: the Childrens' Hospital of Philadelphia Experience, 1974-1994. J Pediatr 1997;131:678-682.

7. Chen YC, et al. Laryngeal neurofibroma: case report of a child. Int J Pediatr Otorhinolaryngol 2002;65:167-170.

8. Ejnell H, Jarund M, Bailey M, et al. Airway obstruction in children due to plexiform neurofibroma of the larynx. J Laryngol and Otol 1996 Nov;110:1065-1068.

9. Suppance JS, Quenelle DJ, Crissman J. Endolaryngeal Neurofibromas. Otolaryngol Head Neck Surg 1980;88:74-78.

\section{ABOUT THE AUTHORS}

\section{Nupur Kapoor Nerurkar}

Consultant Laryngologist, Department of ENT, Bombay Hospital Mumbai, Maharashtra, India

Correspondence Address: D-603, Simla House, Napean Sea Road Mumbai-400036, Maharashtra, India, e-mail: nupurkapoor@yahoo.com

\section{Gauri Kapre}

Clinical Assistant, Department of ENT, Bombay Hospital, Mumbai Maharashtra, India 